\title{
Bax is involved in the anticancer activity of Velcade in colorectal cancer
}

\author{
LIYA SU ${ }^{1}$, QIMUGE SUYILA ${ }^{1}$, LING YANG ${ }^{1}$, HONG LI $^{2}$, YAGUANG XI ${ }^{3}$ and XIULAN SU ${ }^{1}$ \\ ${ }^{1}$ Clinical Medical Research Center of The Affiliated Hospital; ${ }^{2}$ Department of Oncology of The Affiliated People's Hospital, \\ Inner Mongolia Medical University, Hohhot, Inner Mongolia 010050, P.R. China; ${ }^{3}$ Department of Genetics and \\ Stanley S. Scott Cancer Center, Louisiana State University Health Sciences Center, New Orleans, LA 70112, USA
}

Received September 19, 2016; Accepted June 8, 2017

DOI: $10.3892 /$ etm.2017.4857

\begin{abstract}
Numerous chemotherapeutic agents promote tumor cell death by activating the intrinsic apoptosis signaling pathway. This pathway is regulated by mitochondrial dysfunction, which occurs through an intricate process controlled by complex interactions between B-cell lymphoma 2 (Bcl-2) family members and other cellular proteins. Bcl-2-associated $\mathrm{X}$ protein (Bax) is a proapoptotic protein that is an essential component of the intrinsic apoptosis signaling pathway. Patients lacking Bax may be less sensitive to chemotherapy due to an impaired intrinsic apoptosis signaling pathway. The present study demonstrated that Bax expression in colorectal cancer (CRC) tissues was typically increased compared with that in adjacent normal tissues. Furthermore, Bax $^{-/-}$HCT-116 cells exhibited reduced proliferation and colony formation ability compared with $\mathrm{Bax}^{+/+}$HCT116 cells, although the rate of apoptosis of these cells remained unchanged. However, $\mathrm{Bax}^{-/-}$HCT116 cells became more resistant to apoptosis when treated with Velcade. The results of the present study provide novel insights into the relevance of Bax expression to the prognosis of CRC.
\end{abstract}

\section{Introduction}

Colorectal cancer (CRC) remains one of the leading types of cancer in humans, causing $>550,000$ mortalities annually in the United States (1). In addition, the incidence of CRC has increased in Eastern and Western countries over the past decades (2). To improve the survival of patients with CRC, personalized therapy targeting reliable biomarkers is required. B-cell lymphoma 2 (Bcl-2)-associated X protein (Bax), a

Correspondence to: Dr Liya Su or Professor Xiulan Su, Clinical Medical Research Center of The Affiliated Hospital, Inner Mongolia Medical University, 1 Tongdao North Street, Hohhot, Inner Mongolia 010050, P.R. China

E-mail: suliya2307@hotmail.com

E-mail: xlsu2014@163.com

Key words: colorectal cancer, Velcade, Bax, apoptosis proapoptotic protein belonging to the Bcl-2 family is one of the most promising apoptosis-associated biomarkers in several types of human cancers. Bax is an essential component of the intrinsic apoptosis signaling pathway. Patients lacking Bax may be less sensitive to chemotherapy due to an impaired intrinsic apoptosis signaling pathway (3). In CRC, Bax is positively correlated with improved survival outcomes (4,5). However, a previous study reported that the dysregulation of Bax could lead to the resistance of human CRC cells to treatment with sulindac, an effective nonsteroidal anti-inflammatory drug (NSAID) with a chemopreventive effect on CRC (6). Thus far, the use of Bax as a biomarker to aid in clinical decision-making has not been substantiated. Further studies should focus on elucidating the significance of Bax on the apoptosis of CRC cells in response to chemotherapy.

Velcade, also known as bortezomib, is a specific and selective inhibitor of the $26 \mathrm{~S}$ proteasome (7). Velcade was the first proteasome inhibitor approved by the US Food and Drug Administration for the treatment of relapsed or refractory multiple myeloma and mantle cell lymphoma (8). Similar to other proteasome inhibitors, Velcade can stabilize several proteins by inhibiting their degradation; these proteins include transcriptional factors (proto-oncogenes c-Myc, c-Fos and c-Jun), p21, cyclin-dependent kinase inhibitor p27, cellular tumor antigen p53 (p53), nuclear factor $\kappa \mathrm{B}$ inhibitor $\alpha$, cyclins and Bcl-2 family members (Bcl-2 homologous antagonist/killer and Bax) $(9,10)$. Additionally, Velcade can induce the apoptosis of tumor cells through the intrinsic mitochondrial, extrinsic death receptor and endoplasmic reticulum stress response apoptosis signaling pathways (11-13). Clinical studies have revealed that Velcade exhibits limited efficacy against solid tumors when used as a single agent (14-18). However, reports from phase I/II clinical trials have indicated that when combined with other chemotherapy drugs Velcade can significantly sensitize tumor cells to chemotherapy (19). The combination of Velcade with oxaliplatin, leucovorin and 5-fluorouracil as a treatment for CRC has previously been studied $(20,21)$. One clinical trial revealed that 5 of 13 patients had a partial response to Velcade (21). Further trials are required to verify the effectiveness of Velcade. Furthermore, the underlying molecular mechanisms of this regimen remain unclear.

The present study revealed that Bax expression was increased in CRC specimens compared with adjacent normal 
tissues. Bax ${ }^{-/}$HCT116 cells revealed slow growth and modest colony formation ability. When treated with Velcade, the $\mathrm{Bax}^{-/}$ HCT116 cells demonstrated a higher resistance to apoptosis compared with $\mathrm{Bax}^{+/+} \mathrm{HCT} 116$ cells. These results indicate that Bax is a potential marker for predicting the response of patients with CRC to Velcade therapy. In addition, these results suggest that Bax is associated with the anticancer activity of Velcade in CRC.

\section{Materials and methods}

Tissue samples. In total, 13 pairs of CRC and adjacent normal tissue samples (intraoperative tissue was snap-frozen in liquid nitrogen, at $-196^{\circ} \mathrm{C}$ ) were collected from patients with CRC after resection surgery at the Inner Mongolia Medical University Hospital (Hohhot, Inner Mongolia, China) between July and November 2008. The patients selected for the present study were aged between 39 and 76 (8 males and 5 females), did not receive prior radiotherapy or cytotoxic chemotherapy, and were histologically confirmed with colorectal adenocarcinoma. Patients that had received prior radiotherapy or cytotoxic chemotherapy were excluded. All sample collection and handling processes were conducted in accordance with the National Institutes of Health's guidelines and regulations. All participants were verbally informed about the purpose of the present study and provided written informed consent. The present study was approved by the Ethics Committee of the Affiliated Hospital of Inner Mongolia Medical University.

Materials. HCT116 cells were purchased from the American Type Culture Collection (Manassas, VA, USA), and were cultured in Dulbecco's modified Eagle's medium (DMEM; Gibco; Thermo Fisher Scientific, Inc., Waltham, MA, USA) containing $10 \%$ fetal bovine serum (FBS; Gibco; Thermo Fisher Scientific, Inc.) at $37^{\circ} \mathrm{C}$ in a humidified atmosphere containing 5\% $\mathrm{CO}_{2}$. $\mathrm{Bax}^{-/} \mathrm{HCT} 116$ cells were established and characterized according to a previously published protocol (6). Antibodies directed against Bax (cat. no. 2772, 1:1,000 dilution), poly ADP-ribose polymerase (PARP; cat. no. 9542; 1:1,000 dilution), Bcl-2 (cat. no. 2872; 1:1,000 dilution), p53 (cat. no. 9282; 1:1,000 dilution) and $\beta$-actin (cat. no. 4970; 1:10,000 dilution) were purchased from Cell Signaling Technology, Inc. (Danvers, MA, USA). Velcade was purchased from Sigma-Aldrich (Merck KGaA, Darmstadt, Germany).

Cell proliferation assay. The cell proliferation rate was measured using the cell counting kit-8 (CCK-8) assay (Dojindo Molecular Technologies Inc., Rockville, MD, USA) according to the manufacturer's protocol. $\mathrm{Bax}^{+/+}$and $\mathrm{Bax}^{-/-} \mathrm{HCT} 116$ cells were seeded into 96-well plates at a density of 2,000 cells/well. Once the cells had attached to the wells, the appropriate reagents were added and cell proliferation was assessed by determining the absorbance of each well at a wavelength of $450 \mathrm{~nm}$ over 6 days using a microplate reader. Data are presented as the mean \pm standard deviation (SD) of at least three wells from each group. Each experiment was performed in triplicate.

Clonogenic assay. A clonogenic assay was performed as described previously (22). Cells from each group were seeded

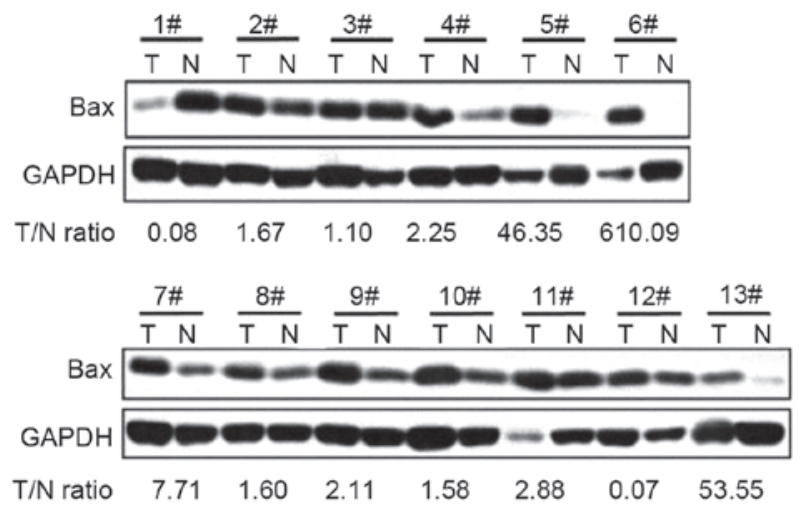

Figure 1. Differential expression of Bax protein in human CRC tissues. The protein level of Bax in 13 pairs of fresh frozen CRC and adjacent normal tissues was examined by western blotting. CRC, colorectal cancer; N, normal tissue; T, tumor tissue; Bax, B-cell lymphoma 2-associated X protein.

into 6-well plates in triplicate at a density of 400 cells/well and cultured for $\leq 3$ weeks until visible colonies had formed. $\mathrm{Bax}^{+/+}$and $\mathrm{Bax}^{-/-}$HCT116 cells reached $80 \%$ confluence were treated with 50 or $100 \mathrm{nM}$ Velcade for $>10$ days until visible colonies had formed. The cell colonies were fixed by methanol for $15 \mathrm{~min}$ at room temperature and stained with a solution containing $0.5 \%$ crystal violet and $25 \%$ methanol for $30 \mathrm{~min}$ at room temperature and washed with tap water to remove excess dye. The number of colonies was counted under a light microscope at room temperature.

Western blot analysis. $\mathrm{Bax}^{+/+}$and $\mathrm{Bax}^{-/-} \mathrm{HCT} 116$ cells were treated with different doses of Velcade $(0,0.2,1$ and $2 \mathrm{nM})$ for $12 \mathrm{~h}$ or treated with $1 \mathrm{nM}$ Velcade for $0,6,12$ and $24 \mathrm{~h}$. Cells were harvested and lysed with 1X SDS lysis buffer [50 mM Tris- $\mathrm{HCl}$ (pH 6.8), $10 \%$ glycerol and 2\% SDS] to extract the total protein. Following the quantification of protein concentration using a Bicinchoninic Acid Protein assay kit (Pierce; Thermo Fisher Scientific, Inc.), $20 \mu \mathrm{g}$ of protein from each sample were separated by SDS-PAGE on a $10 \%$ gel and transferred onto a polyvinylidene difluoride membrane. The membranes were blocked in $5 \%$ non-fat milk for $2 \mathrm{~h}$ at room temperature and then incubated at $4{ }^{\circ} \mathrm{C}$ overnight with primary antibodies. The membranes were washed three times with Tris-buffered saline containing $0.5 \%$ Tween-20 (TBST). The membranes were then incubated with horseradish peroxidase-conjugated secondary antibodies (cat. no. SC-2357; 1:10,000 dilution; Santa Cruz Biotechnology, Santa Cruz, CA, USA) for $1 \mathrm{~h}$ at room temperature and washed with TBST again. Finally, target protein bands were visualized using an Enhanced Chemiluminescence Detection system (Engreen Biosystem, Ltd., Beijing, China). GelQuant.NET software version 1.7.8 (biochemlabsolutions.com/GelQuantNET.html) was used for quantification of the intensity of each band. The expression ratio in tumor $(\mathrm{T})$ vs. normal $(\mathrm{N})$ tissue was calculated using the following formula: $\left(\mathrm{T}_{\mathrm{Bax}} / \mathrm{T}_{\mathrm{GAPDH}}\right) /\left(\mathrm{N}_{\mathrm{Bax}} / \mathrm{N}_{\mathrm{GAPDH}}\right)$.

Apoptosis assay. $\mathrm{Bax}^{+/+}$and $\mathrm{Bax}^{-/-} \mathrm{HCT} 116$ cells were treated with Velcade $(0,10,50$ and $100 \mathrm{nM})$ for $12 \mathrm{~h}$ at $37^{\circ} \mathrm{C}$. The rate of apoptosis was then quantified using a fluorescein isothiocyanate-labeled Annexin V/propidium iodide apoptosis kit (Invitrogen; Thermo Fisher Scientific, Inc.) according to 
A

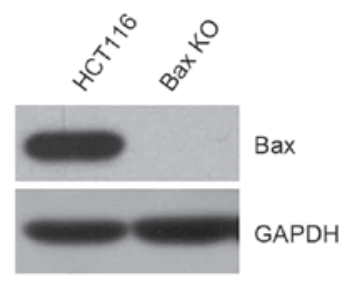

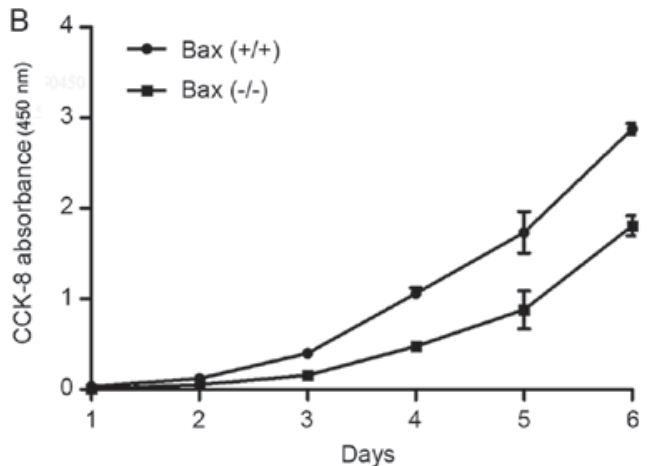

3

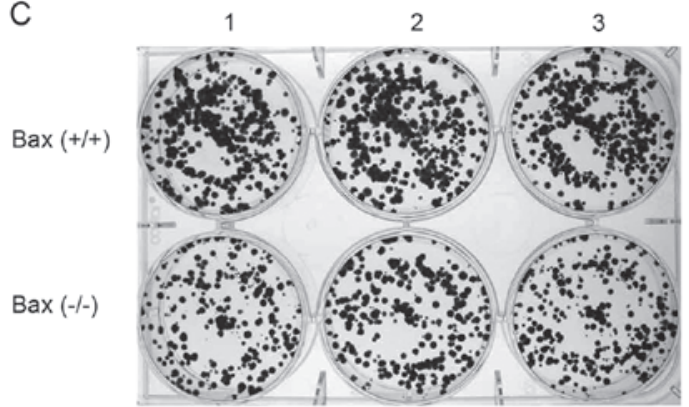

D

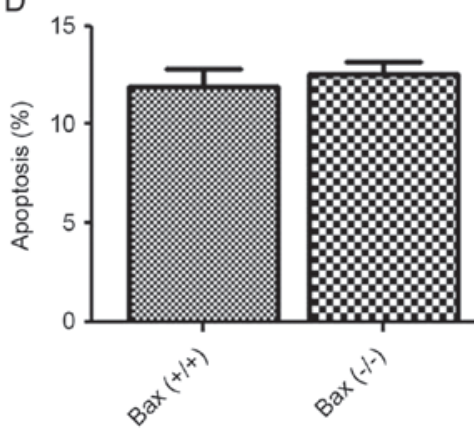

Figure 2. Deletion of Bax in HCT116 cells inhibits cell growth and colony formation. (A) Genetic knockout of Bax in HCT116 cells was verified by western blot analysis. (B) The proliferation of HCT116 cells with or without Bax was examined using the CCK-8 assay. (C) Results of the clonogenic assay in HCT116 cells with or without Bax. (D) Flow cytometry analysis was performed to evaluate the apoptosis of HCT116 cells with or without Bax. Bax, B-cell lymphoma 2-associated X protein; KO, knockout; CCK-8, Cell Counting Kit-8.

the manufacturer's protocol using a FACS Calibur cytometer (BD Biosciences, San Diego, CA, USA).

Statistical analysis. Data are presented as the mean \pm SD. The Student's t-test was used to compare the statistical significance of differences between the mean values of two groups. One-way analysis of variance and the Bonferroni method were employed when there were more than three test groups. Each experiment was performed in triplicate. $\mathrm{P}<0.05$ was considered to indicate a statistically significant difference. All the statistical analysis was performed using the SPSS software 13.0 (SPSS, Inc., Chicago, IL, USA).

\section{Results}

Differential expression of Bax protein in human CRC tissues. The expression level of Bax protein was examined in 13 pairs of fresh frozen CRC and adjacent normal tissues by western blotting. Bax was identified to be differentially expressed in CRC tissue compared with normal colorectal tissue (Fig. 1). The expression of the Bax protein was increased in the majority of the CRC tissues compared with that in the corresponding normal tissues $(11 / 13,85 \%)$. Therefore, decreasing the expression of Bax may be beneficial in inhibiting CRC growth.

Deletion of Bax in HCT116 cells inhibits cell growth and colony formation. To investigate the function of Bax in CRC progression, $\mathrm{Bax}^{-/}$HCT116 cells were established and western blot analysis was performed for verification (Fig. 2A). The effect of Bax on cell proliferation was then evaluated using CCK- 8 and colony formation assays. The results demonstrated that the absence of Bax suppressed the proliferation of HCT116 cells (Fig. 2B and C). However, the knockout of Bax did not affect the rate of apoptosis (Fig. 2D).

Deletion of Bax confers HCT116 cells with resistance to Velcade-induced apoptosis. The downregulation of Bax may enhance the resistance of tumor cells to Velcade (6). Thus, the rate of apoptosis in $\mathrm{Bax}^{-/-}$HCT116 cells was examined following exposure to Velcade, a commonly used drug for the treatment of patients with progressive myeloma. The effect of increasing amounts of Velcade on the rate of apoptosis was measured using flow cytometry. The results revealed that the apoptosis rate of $\mathrm{Bax}^{+/+}$and $\mathrm{Bax}^{-/-} \mathrm{HCT} 116$ cells markedly increased with increasing doses of Velcade (Fig. 3A). However, the percentage of $\mathrm{Bax}^{-/}$HCT116 cells that underwent apoptosis was lower compared with that of $\mathrm{Bax}^{+/+}$HCT116 cells at each Velcade dose. Notably, the difference between $\mathrm{Bax}^{+/+}$HCT116 cells at 50 and $100 \mathrm{~nm}$ of Velcade was statistically significant $(\mathrm{P}<0.05$; Fig. 3A). This indicates that the absence of Bax may increase the resistance of HCT116 cells to Velcade. To validate this phenotype, the expression of an indicator of apoptosis, cleaved PARP, was examined. Cleavage of PARP was reduced in $\mathrm{Bax}^{-/-}$HCT116 cells when exposed to Velcade (Fig. 3B and $\mathrm{C}$ ), indicating that Bax deletion reduces Velcade-induced HCT116 cell apoptosis.

Deletion of Bax increases the resistance of HCT116 cells to Velcade. Considering that the absence of Bax suppressed the proliferation of HCT116 cells, the effect of Bax on HCT116 cell proliferation was evaluated using a colony formation assay. The results revealed that the deletion of Bax significantly 
A

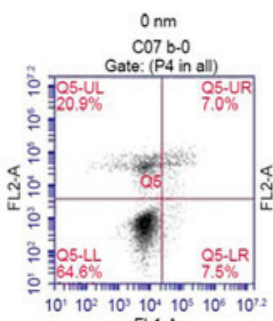

FL1-A

FOB 116-0

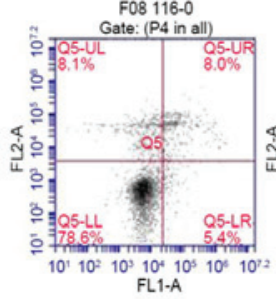

B

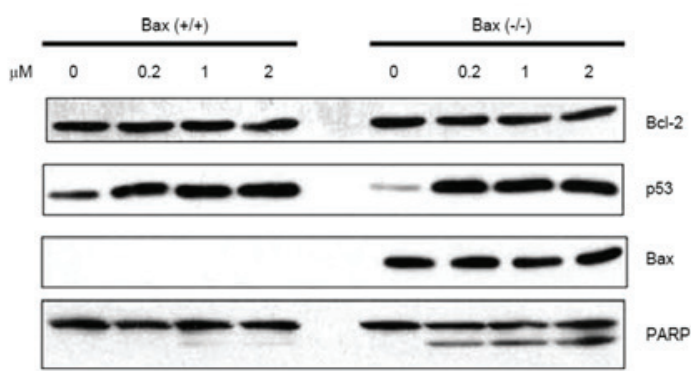

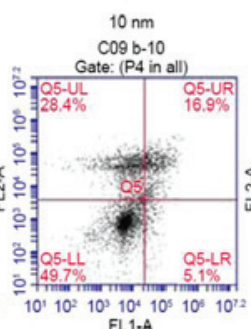

FL1.A

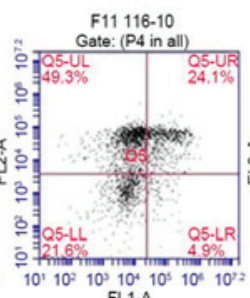

FL 1 -A
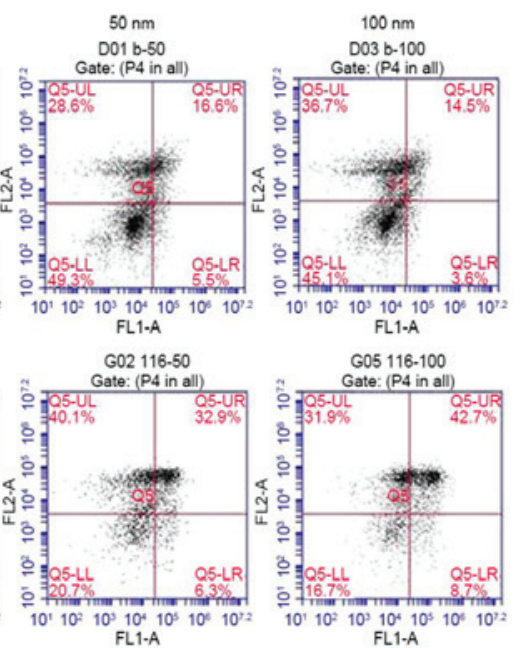

C

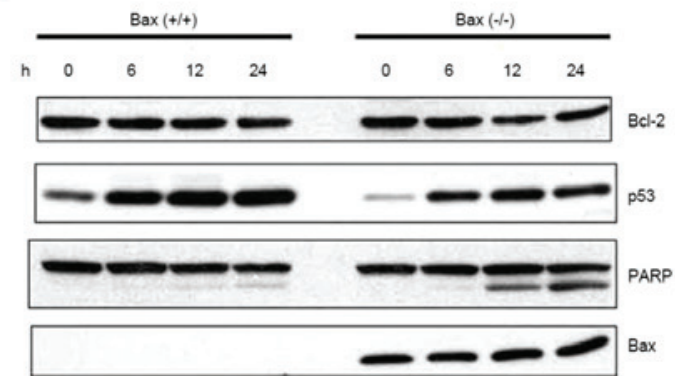

Figure 3. Deletion of Bax confers HCT116 cells with resistance to Velcade-induced apoptosis. (A) Bax ${ }^{+/+}$and Bax ${ }^{-/}$HCT116 cells were incubated with different doses of Velcade $(0,10,50$ and $100 \mathrm{nM})$ for $12 \mathrm{~h}$ and then flow cytometry analysis was performed to evaluate apoptosis. (B) Bax ${ }^{+/+}$and Bax $\mathrm{Ba}^{-/-} \mathrm{HCT}_{116}$ cells were treated with different doses of Velcade $(0,0.2,1$ and $2 \mathrm{nM})$ for $12 \mathrm{~h}$ and then the level of Bax protein was examined via western blotting. $(\mathrm{C}) \mathrm{Bax}{ }^{+/+}$ and $\mathrm{Bax}^{-/} \mathrm{HCT} 116$ cells were treated with $1 \mathrm{nM}$ Velcade for $0-24 \mathrm{~h}$ prior to western blot analysis of Bax expression. ${ }^{*} \mathrm{P}<0.05$ vs. the Bax ${ }^{-/}$group. Bax, B-cell lymphoma 2-associated X protein.
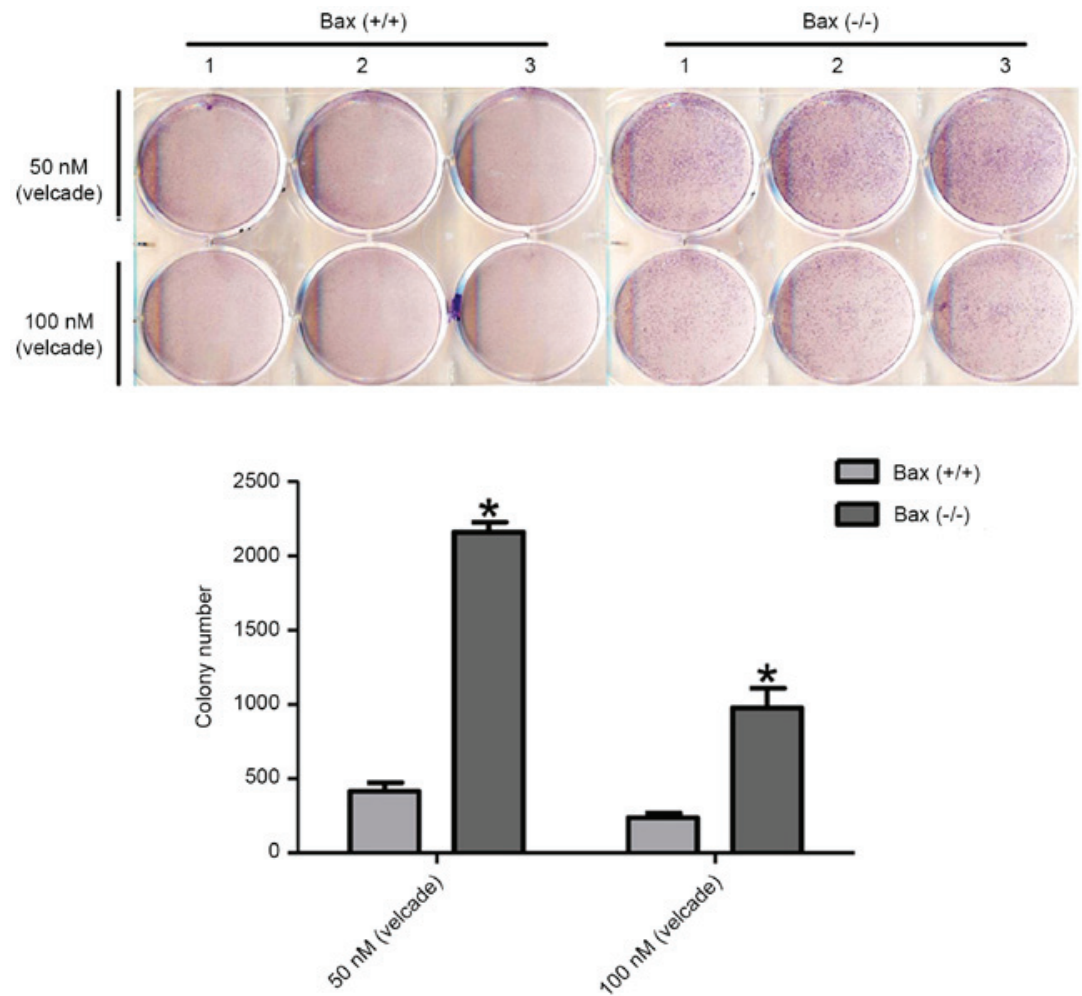

Figure 4. Deletion of Bax increases the resistance of HCT116 cells to Velcade. HCT116 cells with or without Bax were treated with 50 or $100 \mathrm{nM}$ Velcade in triplicate and cultured until visible colonies had formed. The colonies were then stained with crystal violet and images were captured. The colonies were subsequently counted. ${ }^{*} \mathrm{P}<0.05$ vs. the $\mathrm{Bax}^{+/+}$group. Bax, B-cell lymphoma 2 -associated X protein. 
enhanced the resistance of HCT116 cells to Velcade compared with the $\mathrm{Bax}^{+/+} \mathrm{HCT} 116$ cells $(\mathrm{P}<0.05$; Fig. 4).

\section{Discussion}

To date, few biomarkers for predicting the response of patients with CRC to chemotherapy have been identified, which has limited the development of personalized medicine for the treatment of CRC. The association between Bax and the prognosis and response of patients with CRC to surgery and chemotherapy has been analyzed in a series of prospective studies $(23,24)$. Bax expression was identified to be correlated with improved survival outcomes in patients with CRC, indicating that Bax is a promising biomarker for CRC prognosis and positively affects the response of patients to chemotherapy (25). Another study demonstrated that in cultured $\mathrm{CRC}$ cells the absence of Bax reduced the apoptotic response to chemopreventive agents, including NSAIDs (6).

In the present study, Bax expression was examined in paired CRC and adjacent normal tissues. Bax was identified to be aberrantly expressed CRC compared with normal tissues. In addition, $\mathrm{Bax}^{-/-}$HCT116 cells demonstrated reduced proliferation and colony formation. When treated with Velcade, Bax $^{-/}$HCT116 cells exhibited increased resistance to Velcade compared with $\mathrm{Bax}^{+/+} \mathrm{HCT} 116$ cells. These results indicate that Bax is involved in Velcade-induced apoptosis in CRC cells and is a novel biomarker for the prognosis of patients with CRC. In addition, the role of Bax in the anticancer activity of Velcade highlights the potential use of Velcade as an adjuvant and/or neoadjuvant therapy for patients with CRC.

\section{Acknowledgements}

The present study was supported by the Inner Mongolia Natural Science Foundation (grant no. 2011BS1117) and the Inner Mongolia Medical University Science and Technology Million Project (grant no. NY2011BW005).

\section{References}

1. Walther A, Johnstone E, Swanton C, Midgley R, Tomlinson I and Kerr D: Genetic prognostic and predictive markers in colorectal cancer. Nat Rev Cancer 9: 489-499, 2009.

2. Bretthauer M: Colorectal cancer screening. J Intern Med 270: 87-98, 2011.

3. Ierano C, Chakraborty AR, Nicolae A, Bahr JC, Zhan Z, Pittaluga S, Bates SE and Robey RW: Loss of the proteins Bak and Bax prevents apoptosis mediated by histone deacetylase inhibitors. Cell Cycle 12: 2829-2838, 2013.

4. Pietrantonio F, Biondani P, Ciurlia E, Fanetti G, Tessari A, Bertarelli G, Bossi I, Musella V, Melotti F, Di Bartolomeo M, et al: Role of BAX for outcome prediction in gastrointestinal malignancies. Med Oncol 30: 610, 2013.

5. Hector S and Prehn JH: Apoptosis signaling proteins as prognostic biomarkers in colorectal cancer: A review. Biochim Biophys Acta 1795: 117-129, 2009.

6. Zhang L, Yu J, Park BH, Kinzler KW and Vogelstein B: Role of BAX in the apoptotic response to anticancer agents. Science 290: 989-992, 2000.

7. Adams J, Palombella VJ, Sausville EA, Johnson J, Destree A, Lazarus DD, Maas J, Pien CS, Prakash S and Elliott PJ: Proteasome inhibitors: A novel class of potent and effective antitumor agents. Cancer Res 59: 2615-2622, 1999.

8. Buac D, Shen M, Schmitt S, Kona FR, Deshmukh R, Zhang Z, Neslund-Dudas C, Mitra B and Dou QP: From bortezomib to other inhibitors of the proteasome and beyond. Curr Pharm Des 19: 4025-4038, 2013.
9. Pei XY, Dai Y and Grant S: The proteasome inhibitor bortezomib promotes mitochondrial injury and apoptosis induced by the small molecule Bcl-2 inhibitor HA14-1 in multiple myeloma cells. Leukemia 17: 2036-2045, 2003.

10. Lara PN Jr, Davies AM, Mack PC, Mortenson MM, Bold RJ, Gumerlock PH and Gandara DR: Proteasome inhibition with PS-341 (bortezomib) in lung cancer therapy. Semin Oncol 31 (1 Suppl 1): 40-46, 2004

11. Nencioni A, Grunebach F, Patrone F, Ballestrero A and Brossart P: Proteasome inhibitors: Antitumor effects and beyond. Leukemia 21: 30-36, 2007.

12. Lee AH, Iwakoshi NN, Anderson KC and Glimcher LH: Proteasome inhibitors disrupt the unfolded protein response in myeloma cells. Proc Natl Acad Sci USA 100: 9946-9951, 2003.

13. Mitsiades N, Mitsiades CS, Poulaki V, Chauhan D, Fanourakis G, Gu X, Bailey C, Joseph M, Libermann TA, Treon SP, et al: Molecular sequelae of proteasome inhibition in human multiple myeloma cells. Proc Natl Acad Sci USA 99: 14374-14379, 2002.

14. Blaney SM, Bernstein M, Neville K, Ginsberg J, Kitchen B, Horton T, Berg SL, Krailo M and Adamson PC: Phase I study of the proteasome inhibitor bortezomib in pediatric patients with refractory solid tumors: A Children's Oncology Group Study (ADVL0015). J Clin Oncol 22: 4804-4809, 2004.

15. Papandreou CN, Daliani DD, Nix D, Yang H, Madden T, Wang X, Pien CS, Millikan RE, Tu SM, Pagliaro L, et al: Phase I trial of the proteasome inhibitor bortezomib in patients with advanced solid tumors with observations in androgen-independent prostate cancer. J Clin Oncol 22: 2108-2121, 2004.

16. Shah MH, Young D, Kindler HL, Webb I, Kleiber B, Wright J and Grever M: Phase II study of the proteasome inhibitor bortezomib (PS-341) in patients with metastatic neuroendocrine tumors. Clin Cancer Res 10: 6111-6118, 2004.

17. Li T, Ho L, Piperdi B, Elrafei T, Camacho FJ, Rigas JR, PerezSoler R and Gucalp R: Phase II study of the proteasome inhibitor bortezomib (PS-341, Velcade) in chemotherapy-naïve patients with advanced stage non-small cell lung cancer (NSCLC). Lung cancer 68: 89-93, 2010.

18. Yang CH, Gonzalez-Angulo AM, Reuben JM, Booser DJ, Pusztai L, Krishnamurthy S, Esseltine D, Stec J, Broglio KR, Islam R, et al: Bortezomib (VELCADE) in metastatic breast cancer: Pharmacodynamics, biological effects and prediction of clinical benefits. Ann Oncol 17: 813-817, 2006.

19. Milano A, Perri F and Caponigro F: The ubiquitin-proteasome system as a molecular target in solid tumors: An update on bortezomib. OncoTargets Ther 2: 171-178, 2009.

20. O'Neil BH, Raftery L, Calvo BF, Chakravarthy AB, Ivanova A, Myers MO, Kim HJ, Chan E, Wise PE, Caskey LS, et al: A phase I study of bortezomib in combination with standard 5-fluorouracil and external-beam radiation therapy for the treatment of locally advanced or metastatic rectal cancer. Clin Colorectal Cancer 9: 119-125, 2010.

21. Caponigro F, Lacombe D, Twelves C, Bauer J, Govaerts AS, Marréaud S, Milano A and Anthoney A: An EORTC phase I study of Bortezomib in combination with oxaliplatin, leucovorin and 5-fluorouracil in patients with advanced colorectal cancer. Eur J Cancer 45: 48-55, 2009.

22. Huang Z, Li S, Song W, Li X, Li Q, Zhang Z, Han Y, Zhang X, Miao S, Du R and Wang L: Lysine-specific demethylase 1 (LSD1/KDM1A) contributes to colorectal tumorigenesis via activation of the Wnt/ $\beta$-catenin pathway by down-regulating dickkopf- 1 (DKK1) [corrected]. PloS One 8: e70077, 2013.

23. Katkoori VR, Suarez-Cuervo C, Shanmugam C, Jhala NC, Callens T, Messiaen L, Posey J III, Bumpers HL, Meleth S, Grizzle WE and Manne U: Bax expression is a candidate prognostic and predictive marker of colorectal cancer. J Gastrointest Oncol 1: 76-89, 2010.

24. Sturm I, Köhne CH, Wolff G, Petrowsky H, Hillebrand T, Hauptmann S, Lorenz M, Dörken B and Daniel PT: Analysis of the $\mathrm{p} 53 / \mathrm{BAX}$ pathway in colorectal cancer: Low BAX is a negative prognostic factor in patients with resected liver metastases. J Clin Oncol 17: 1364-1374, 1999.

25. Chen YJ and Chung V: Challenges toward personalized treatment of localized colorectal cancer. J Gastrointest Oncol 1: 74-75, 2010. 